are functions of $\left(\xi_{1}, \xi_{2}, \xi_{3}\right)$ and

$$
A=A_{q} \xi_{q}=a_{p q} \xi_{p} \xi_{q} .
$$

To get the force acting on the particle, one can surround the particle by a sphere of radius $r$ and center at the origin, and calculate the stress on the sphere as $r \rightarrow m / 2$. Using the gravitational stress-energy pseudotensor ${ }^{7} t_{\mu}{ }^{\nu}$, one finds that the stress acting across the surface of the sphere has components

$$
\mathfrak{t}_{k}{ }^{p} \xi_{p}=-\left(1 / 4 \pi m^{2}\right) \xi_{k}\left[8+m\left(a_{p p}-a_{p q} \xi_{p} \xi_{q}\right)\right]
$$

for $r=m / 2$. Using (20), one gets

$$
\mathfrak{t}_{k}^{p} \xi_{p}=-\left(2 / \pi m^{2}\right) \xi_{k} \text {. }
$$

Since the integral of (22a) over the sphere vanishes, it follows that the force on the particle vanishes.

This result represents the desired generalization. However, one can try to express it in terms of the external field in which the particle is located. This can be done provided, in Eq. (14), the series in the brackets are convergent for values of $r$ large compared to $m$. In that case, for such values of $r$, these equations become

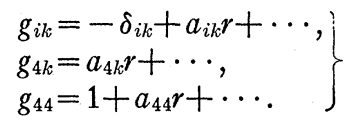

${ }^{7}$ See, for example, reference 6, p. 134.
Now, for $r$ large compared to $m$, one can expect the contribution of the particle to the field to be unimportant, so that Eq. (23) can be considered to describe the external field. If this external field were free from singularities in the absence of the particle, it would be expressed in the form

$$
g_{\mu \nu}=\left(g_{\mu \nu}\right)_{0}+\left(g_{\mu \nu, p}\right)_{0} \xi_{p} r+\cdots,
$$

where the subscript 0 denotes the value at the origin. Comparing with (23) we see that under these conditions it would be necessary to have

$$
a_{\mu \nu}=a_{\mu \nu p} \xi_{p},
$$

where the $a_{\mu \nu p}$ are constants. Going back to (20), we find that with this condition

$$
a_{i k}=a_{44}=0 .
$$

It follows that, for the metric associated with the external field, given by Eq. (23),

$$
\left\{\begin{array}{c}
\lambda \\
44
\end{array}\right\}_{0}=0
$$

which agrees with the equation of the geodesic for a particle at rest.

\title{
Remarks on Intra- and Inter-Molecular Migration of Excitation Energy
}

\author{
JAMES Franck \\ Department of Chemistry, University of Chicago, Chicago, Illinois \\ AND ROBERT LIVINGSTON \\ Department of Chemistry, University of Minnesota, Minneapolis, Minnesota
}

$\mathbf{M}$ IGRATION of excitation energy between molecules or inside a molecule from one of its groups to another is observed under many different conditions. The transferred excitation energy may be used just as the directly absorbed light energy for the promotion of chemical processes, or it may be dissipated into heat. The most remarkable facts are that the excitation energy under suitable conditions can be transferred with a high yield over distances of $\sim 50-100 \mathrm{~A}$ and that molecules or molecular groups which do not possess the quality to fluoresce still are able to transfer their excitation energy to energy acceptors which may not be in contact with them. There is no doubt that every example of transfer of electronic excitation energy observed so far can be understood either by an electron migration, or by exciton migration, or by the process of sensitized fluorescence, but in many cases uncertainty exists as to whether one of these three processes is solely responsible for a particular phenomenon and, if so, which one it is. In other cases experiences with migration of excitation energy are indiscriminately used as a proof for migration of energy in general. Especially, interpretations of energy transfer by and in substances of biological importance are often based on doubtful hypotheses. The present paper is a brief survey of the principles of the three processes by which migration of excitation energy can occur and gives examples of how a distinction between them can be reached.

Excitation energy in insulators may be transferred by electrons which by an act of light absorption are lifted 
into a conductivity band where they can travel. The energy will be released (or partially released) if the electron encounters on its way a potential well; for instance, a positive hole with which it recombines. The recombination energy may, according to conditions, be emitted as light, transformed into heat movement, or be transferred to a suitable acceptor. In the process of fluorescence and phosphorescence emission of certain impurities in ionic crystals, the transfer of energy by electrons moving in the conduction band plays a decisive role. However, there are indications that the last step in the transfer of excitation energy to the impurity is achieved by sensitized fluorescence. The non-fluorescent recombination of an electron with a positive hole, which in itself does not give observable light emission, seems to be able to transfer its energy to the impurity. ${ }^{1}$

The presence of a free electron in the crystal lattice causes disturbances. It attracts positive centers and repels negative ones. Under these conditions migration is only possible if the electron stays in the neighborhood of an ion for a time which is much shorter than an oscillation period of the building stones of the lattice. Expressed in the language of quantum mechanics, the electron belongs to the whole lattice. The smaller the disturbances of the lattice by temperature movement and by imperfections, the greater is the distance an electron can migrate without being trapped.

Similarly if a complex molecule having an extended system of conjugated double bonds absorbs light, the excitation belongs to the conjugated system as a whole, and can therefore be used for photo-chemical changes in all parts of that system.

Exciton movement is the name given by Frenkel to a quick movement of excitation energy in crystals through a great number of building stones of the crystal. Again the movement is so quick that the energy stays in a particular atom or group of atoms for a much shorter time than the duration of an oscillation. (The theory was developed by Frenkel, and especially by Peierls; see also Franck and Teller. ${ }^{2}$ ) That implies a strong coupling of the resonating build-stones in the crystal, and correspondingly the spectrum will look quite different from that of the gas. There is only one group of observations known which very probably has to be explained by exciton migration. These are absorption and fluorescence phenomena observed in dyestuff polymers of pseudo-isocyanines. As Jelley, Scheibe, Sheppard, and others ${ }^{3}$ have shown, thousands of these

\footnotetext{
1 J. Franck, Ann. d. Physik 3, 62 (1948).

2 J. Frenkel, Phys. Rev. 37, 17, 1276 (1931); Physik. Zeits. Sowjetunion 9, 158 (1936). R. Peierls, Ann. d. Physik 13, 905 (1932). J. Franck and E. Teller, J. Chem. Phys. 6, 861 (1938).

3 E. E. Jelley, Nature 138, 1009 (1936); 139, 631 (1937). G. Scheibe, Zeits. f. angew. Chemie 50, 51 (1937); 52, 631 (1939). Scheibe, Kandler, and Echer, Naturwiss. 25, 75 (1937). Scheibe, Mareis, and Echer, Naturwiss. 25, 474 (1937). Scheibe, Schöntag, and Katheder, Naturwiss. 27, 499 (1939). O. Fischer and G. Scheibe, J. prakt. Chem. 50, 212 (1937). S. E. Sheppard, Rev. Mod. Phys. 14, 303 (1942). R. W. Mattoon, J. Chem. Phys. 12, 268 (1944).
}

dye molecules can be linked together by water molecules. The monomers show broad absorption bands in the visible and no fluorescence. Polymerization shifts somewhat the maxima of the molecular absorption bands and in addition a new, narrow, and very strong absorption band (comparable to a broadened spectral line) appears. Absorption of light in the two molecular bands, as well as absorption in the new band, causes strong fluorescence emission of light with the wavelengths of the narrow band. Observations on polarization of the fluorescence and of its extinction by collision with molecules of certain quenching substances indicate that the narrow absorption and emission band is a quantum transition of the polymer as a whole.

Sensitized fluorescence was first observed in monatomic gases. ${ }^{4}$ For instance, mercury vapor excited by absorption of $2537 \mathrm{~A}$ may transfer its energy to other metal vapors present. As a result the resonance fluorescence of mercury will be quenched and the fluorescence of the added metal vapor appears. To achieve the transfer of excitation energy, the metal atoms to be excited must have energy levels lower than the one excited in the mercury atom. The yield of the sensitized fluorescence becomes very great when there is an energy level of practically the same height as the level in the primary excited atom. If this condition of resonance or near resonance exists, energy transfer between atoms can take place over a distance of $\sim 50 \mathrm{~A}$ or even more. (Compare experiments of Cario and Franck, Beutler, Datta, and the theory of Kallmann and London. ${ }^{4}$ )

Sensitized fluorescence should also occur in systems consisting of complex molecules. A molecule, excited by light of wave-length shorter than those absorbed by a second compound which is also present in the system, should be able to transfer its energy to a molecule of the latter type. The efficiency of transfer should be high if the fluorescence spectrum of the primarily excited molecule overlaps the absorption spectrum of the second molecule. This process of sensitized fluorescence ought not to be confused with the trivial process of light emission by the first molecule followed by the absorption of the fluorescent light by the second, since these two processes are, quantitatively, entirely different functions of the concentrations, etc. The occurrence in the vapor phase of the fluorescence of indigo sensitized by aniline, and of aniline sensitized by benzene, has been reported by Prileshajewa and co-workers. ${ }^{5}$ Recently, W. Watson ${ }^{6}$ has observed the fluorescence of chlorophyll $a$ sensitized by chlorophyll $b$ in relatively dilute methanolic solutions.

Sensitized fluorescence is apparently also responsible for the transfer of excitation energy in crystals of

${ }^{4}$ G. Cario and J. Franck, Zeits. f. Physik 17, 202 (1923). H. Beutler and B. Josephy, Naturwiss. 15, 549 (1927). G. L. Datta, Zeits. f. Physik 37, 625 (1926). H. Kallmann and F. London, Zeits. f. physik. Chemie (B) 2, 207 (1928).

${ }^{5}$ N. Prileshajewa, Acta Physicochimica 1, 785 (1935). N. Prileshajewa and A. Klimowa, Acta Physicochimica 7, 163 (1937). A. Terenin, Acta Physichimica 18, 210 (1943).

${ }^{6}$ W. F. Watson (unpublished work), 
anthracene containing naphthacene as an impurity of small concentration and in a number of similar systems. The fluorescence of anthracene is quenched and the naphthacene is excited to light emission. ${ }^{7}$ The transfer cannot be achieved by electron migration because no ionization takes place in the anthracene lattice under the experimental conditions used. Also exciton migration cannot be responsible for the energy transport because the absorption spectrum of the anthracene shows no indication of a strong coupling of the molecules, and the spectral distribution of the fluorescence spectrum indicates that the excited state lasts long enough in every molecule to permit adjustment of atomic oscillation energy to the prevailing temperature; i.e., the time every particular molecule stays excited must be much greater than the duration of one atomic oscillation. That is just the condition which excludes exciton migration and makes sensitized fluorescence possible.

Of special interest is the migration of excitation energy in dye solutions containing only one species of dye molecules at relatively high concentrations. In that case the energy may migrate through many dye molecules before the radiation escapes out of the solution. Neither fluorescence spectrum nor the fluorescence intensity will change by radiationless deexcitations of molecules balanced by the excitation of other molecules of the same type (provided the transitions do not occur too often during the lifetime of the excited state). The migration can be studied by observations of the polarization of the fluorescent light. Fluorescence excited by polarized light is strongly polarized in solvents of high viscosity and at low concentration of the dye. With rising dye concentration, depolarization occurs because the excitation energy will migrate through molecules with random directions of their electronic resonators (Wavilow, Pringsheim, and others. ${ }^{8}$ ) Perrin ${ }^{9}$ developed a theory for this process based on Kallmann's and Rosen's theory of sensitized fluorescence. Recently, Förster ${ }^{10}$ has developed a much improved quantum-mechanical theory which is in good agreement with observations. His main result is that under suitable conditions (high fluorescence yield, strong overlapping of absorption and fluorescence spectrum), the energy may migrate over distances of

7 A. Winterstein and K. Schön, Naturwiss. 22, 237 (1934). E. J. Bowen, Nature 142, 108 (1938); 153, 623 (1944); 159, 706 (1947). E. J. Bowen, J. Chem. Phys. 13, 306 (1945). E. J. Bowen and A. H. Williams, Trans. Faraday Soc. 35, 765 (1939). S. G. Ganguley, Nature 151, 673 (1943); 153, 622 (1944); S. G. Ganguley, J. Chem. Phys. 13, 128 (1945); S. G. Ganguley, Ind. J. Phys. 26, 7 (1943). F. Weigert, Trans. Faraday Soc. 36, 1033 (1940). J. A. Miller and C. A. Baumann, Cancer Res. 3, 217 (1943). K. S. Krishnan and P. K. Seshan, Proc. Ind. Acad. Sci. 8A, 487 (1938).

${ }^{8}$ W. L. Lewschin, Zeits. f. Physik 26, 274 (1924); 34, 330 (1925). P. Pringsheim and I. W. Wavilow, Zeits. f. Physik 37, 705 (1926).

${ }^{9} \mathrm{~F}$. Perrin, Comptes Rendus 180, 581 (1925); 181, 514 (1925);

F. Perrin, J. de phys. et rad. 7, 390 (1926).

${ }_{10}$ T. Förster, Ann. d. Physik 2, 55 (1948).
50-100A. In the case of chlorophyll in a concentration of about $\frac{1}{10}$ molar, the theory gives the result that the energy should move through 10,000 molecules during the natural lifetime of the excited chlorophyll. The lifetime is calculated in the usual way from the absorption spectrum. Since the concentration of the chlorophyll in plants was found to be $\sim \frac{1}{10}$ molar in the grana, ${ }^{11}$ the author regards the result as a confirmation of the theory of the so-called photosynthetic unit. The term, photosynthetic unit, introduced first by Gaffron and Wohl ${ }^{12}$ describes the hypothesis that the energy of light absorption acts, taking place somewhere in a unit of several thousand chlorophyll molecules, always travels to the same spot where one carbon dioxide in contact with a suitable enzyme may use the energy for photosynthetic reduction. Förster's calculation does not, however, confirm this hypothesis because the author seems to be unaware that the fluorescence yield in living plants is only about $1 / 1000$; i.e., the lifetime is one thousand times smaller than the natural one and the excitation, according to Förster's calculations, can move only through 10 molecules. Franck and Teller have pointed out in their paper on exciton movement that if the idea of the photosynthetic unit were right, the energy must move as quickly as an exciton from molecule to molecule. No indications in the spectrum of chlorophyll in plants justify the assumption of strong enough coupling of the chlorophyll molecules for such a quick energy transfer. In the meantime, it was shown that other assumptions ${ }^{13}$ than the photosynthetic unit can be used for the explanation of the observation for which the unit was introduced.

The fluorescence yield of chlorophyll is small not only in plants, but also in solutions of pure hydrocarbons. ${ }^{14}$ In some other organic solvents fluorescence yields as high as $\frac{1}{12}$ are observed if the concentration of the pigment is low. ${ }^{14,15}$ Self-quenching has been observed $^{16}$ for practically all dye solutions which have been studied over a wide enough range of concentrations, but no generally satisfactory explanation has been given. In some cases, ${ }^{17}$ reversible dimerization appears to be reasponsible for self-quenching. However, in the case of chlorophyll the dependence of self-quenching upon concentration and temperature is similar to that expected for the migration of excitation energy, and therefore a direct connection between these two pro-

\footnotetext{
${ }^{11}$ See E. Rabinowitch, Photosynthesis (Interscience Publishers, Inc., New York, 1945), Vol. I, pp. 411-2.

${ }^{12}$ H. Gaffron and K. Wohl, Naturwiss. 24, 81, 103 (1936).

${ }_{13}$ J. Franck and K. F. Herzfeld, J. Phys. Chem. 45, 978 (1941).

${ }_{14} \mathrm{R}$. Livingston, W. Watson, and J. MacArdle, J. Am. Chem. Soc. 71, 1542 (1949).

${ }_{15}$ T. A. Prins, Nature 134, 457 (1934).

${ }^{16} \mathrm{~F}$. Witte, J. Chem. Phys. 26, 276 (1929). J. Bouchard, J. Chem. Phys. 33, 128 (1936). E. J. Bowen and A. H. Williams, Trans. Faraday Soc. 35, 765 (1939). G. Körtum and B. Finckh, Zeits. f. physik. Chemie B52, 263 (1942). S. T. Vavilov and P. P. Feofilov, Doklady Acad. Sci. (URSS) 34, 243 (1942).

${ }_{17}$ E. Rabinowitch and L. F. Epstein, J. Am. Chem. Soc. 63, 69 (1941). W. Lewschin, Acta Physiochimica (URSS) 1, 685 (1935); 2, 221 (1935).
} 
cesses appears probable. If one accepts this point of view, the following considerations may be an explanation: A fluorescence yield smaller than unity observed under conditions where no photo-chemical reaction or photo-ionization can occur is an indication that the lifetime of the excited state is terminated by the process of internal conversion of the electronic excitation energy into oscillation energy of the atomic constituents of the molecule. ${ }^{18}$ This radiationless transition occurs when, by internal movement of the atoms in the excited molecule, a relative position of all atomic constituents is reached which is identical with an atomic constellation in the ground state. In other words, the transition comes to pass at a point which belongs to the polydimensional mountain range of the potential of the excited state as well as to that of the ground state. It always takes some time to reach that point in the potential diagram. If the time is longer than the natural lifetime of the excited molecule, the fluorescence yield becomes unity; if it is very short as compared with the lifetime, the fluorescence yield may become so small that no fluorescence will be observed. However, even in this case one has to expect that a fluorescence emission actually will take place during a time which must be longer than the duration of an atomic oscillation; the time may be of the order of magnitude of about $10^{-12} \mathrm{sec}$. If we use the conventional time of $10^{-8} \mathrm{sec}$. as the natural lifetime, the fluorescence yield may be $10^{-4}$ which is near enough to zero, at least for the observation methods of fluorescence employed hitherto. The spectrum of this postulated fluorescence is expected to cover all wave-lengths longer than the exciting frequency. The well-known effect of quenching the fluorescence by raising the temperature is caused by the fact that the point of internal conversion is reached quicker if the temperature agitation of the oscillatory system of the molecule is strong. Concentration quenching of the fluorescence may then be analogous to temperature quenching. If the excitation energy migrates through many molecules, it will encounter some which by temperature fluctuation contain higher than average energy in some degree of freedom of those oscillations which are responsible for reaching quickly the critical point for internal conversion. That will cause a lowering of the average yield of fluorescence if the energy remains in these particular molecules long enough for internal conversion to occur. More experiments are necessary to test this proposed explanation and to compare it to those specific mechanisms which were suggested by Förster.

The considerations given in the preceding paragraph lead us to the conclusion that light absorption in complex molecules (with the exception of ionizing irradiation) always gives some fluorescence even if it is

${ }^{18}$ E. Teller, J. Phys. Chem. 41, 109 (1937). J. Franck and R. Livingston, J. Chem. Phys. 9, 184 (1941). J. Franck and H. Sponer, Vol. Commemor. Victor Henri, Contrib. Structure Moleculaire (Liège, Belgium, 1948). too weak for observation with the usual methods. The question arises whether a lifetime of $\sim 10^{-12}$ sec. of the excited state of such molecules may be long enough to transfer the excitation energy to suitable acceptors in the neighborhood. This assumption was introduced by one of the present writers to explain certain observations of phosphorescence and fluorescence in ionic lattices. However, after the publication of Förster's theory, it became possible to estimate the order of magnitude of energy transfer which may be expected in these cases. For the reasons mentioned, we assume that the lifetime is about $10^{-4}$ of the natural lifetime. During that time the molecule excited by light absorption can be compared to a resonator which changes its frequency from an upper limit equal to that of the exciting light to very low values. If a complex molecule acts as an acceptor whose absorption spectrum lies on the long wave-length side of that of the primarily excited molecule, the time in which a transition may occur can not be much smaller than $\sim 5 \cdot 10^{-13} \mathrm{sec}$. The frequency of a transfer during this abnormally short lifetime to an energy acceptor will be $2 \cdot 10^{4}$ times smaller than for normal lifetimes. Values calculated by Förster for some dyestuffs with normal lifetimes show that a transfer over a distance of a few angströms will occur very frequently even if the lifetime is shortened by the factor estimated above. Consequently, there is no difficulty explaining observations like the ones made by Weissmann..$^{19} \mathrm{He}$ found that irradiation of europium salicylaldehyde, or similar compounds, with light absorbed in the organic part of the molecule, causes fluorescence of the europium. There is no doubt that the energy, by the process of sensitized fluorescence, is transferred from the part of the primary excitation which is very short-lived, to the europium ion which has a long life during the excited state and is, therefore, a good light emitter. Electron or exciton migration is out of the question in this case.

There are other cases known in which, in proteins, excitation energy is transferred over greater distances, and they have been interpreted by several authors as an indication that proteins have a special quality for energy transmission. ${ }^{20}$ Kubowitz and Haas ${ }^{21}$ have observed that the enzyme urease (which contains a small, so-called prosthetic group in conjunction with a protein molecule of the molecular weight of 450,000 ), can be deactivated by absorption of light in the protein molecule. While the absorption spectrum indicates that in different wave-length regions different electronic transitions are excited in the protein by the light absorption, the quantum yield of inactivation is constant throughout the whole spectrum. Obviously a chemical change is induced in the prosthetic group by a transfer of the excitation energy from the protein molecule to this group which acts as an energy acceptor. The yield, however, is small. It is, according to Jordan, about

\footnotetext{
${ }^{19}$ S. J. Weissmann, J. Chem. Phys. 10, 214 (1942).

20 A. Szent-Györgyi, Nature 148, 157 (1941).

${ }_{21}$ F. Kubowitz and E. Haas, Biochem. Zeits. 257, 337 (1933).
} 
$0.005 .^{22}$ Jordan believes that an electron is lifted by the absorption process into the conduction band of the protein molecule and that the energy is transferred by its migration to the prosthetic group. The present writers regard that hypothesis as very improbable. Neither are there any indications in the absorption spectrum which justify the assumption of the occurrence of an internal photo-effect, nor does the structure of the protein, with its weak linkages in the folds of the polypeptide chains, seem suitable for the occurrence of a conduction band. W. Schmitt ${ }^{23}$ assumes that a perfect resonance of the hydrogen bonds exists between the carbonyl and amide groups of neighboring amino acids and constructs in this way a system of conjugated double bonds stretching throughout the whole protein molecule. While this idea is an intriguing one, it does not seem to be in accord with the fact that the proteins do not absorb visible light, a quality which an extended system of conjugated double bands should possess. Wirt $z^{24}$ assumes that an exciton migration may occur in the protein. Again it may be doubted that that can be the case. Since no fluorescence is observed, the migration through many amino acid groups should, according to that hypothesis, be achieved in $10^{-12}$ sec. or even shorter time. That would, just as it was discussed for the case of the photosynthetic unit, require an exceedingly strong coupling between the amino acid groups, for which the spectrum gives no indication. However, a migration by sensitized fluorescence is possible. A calculation shows that one molecule of urease having a molecular weight of 450,000 will fill the volume of a sphere with the radius of about $60 \mathrm{~A}$. If one prosthetic group is associated with every protein, the concentration of the prosthetic group in concentrated protein is about $1 / 500$ molar. We may estimate approximately the frequency of the energy transfer in that case by taking the value calculated by Förster for chlorophyll at the same concentration and dividing it by $10^{4}$

22 P. Jordan, Naturwiss. 26, 693 (1938).

${ }^{23}$ W. Schmitt, Zeits. f. Naturforschung 2b, 98 (1947)

${ }^{24} \mathrm{~K}$. Wirtz, Zeits. f. Naturforschung 2b, 94 (1947). on account of the short lifetime of the excitation energy in the protein. The calculation gives a quantum yield for transfer of about $1 / 1000$. While this value agrees even better than may be expected from the crude calculation with the observed quantum yield $5 / 1000$, it is by no means an unambiguous proof for our assumption that the transfer in this particular case is caused by the process of sensitized fluorescence. Observations are lacking about the quantum yield of absorption processes taking place in the prosthetic groups themselves. Our calculation infers that in this case the quantum yield should be unity.

A more clear cut example may be taken from observations made by Bücher and Kaspers ${ }^{25}$ with the carbon monoxide-myoglobin complex. Myoglobin is a respiration ferment containing two haemin components as prosthetic groups attached to a protein of the molecular weight of about 30,000 . The iron in the haemins can combine with carbon monoxide; the heat of dissociation is $<15 \mathrm{Kcal}$. If this compound is irradiated with light which is absorbed by the haemin, a splitting off of the carbon monoxide occurs with the quantum yield of unity. Bücher and Kaspers observed that the same process with the same yield can also be achieved by light of shorter wave-length which is not absorbed by the haemin but by the protein. A sufficient part of the energy of every quantum must be transferred to the haemin to cause a dissociation with the yield of unity. A rough calculation similar to that made for the urease shows that the iron concentration corresponds to about $\frac{1}{10}$ mole. If we compare again the energy transfer with that of chlorophyll of equal concentration and divide by $10^{4}$, we find that about one transfer during the lifetime of about $10^{-12}$ should be possible. Again the calculation cannot claim to give more than orders of magnitude. We conclude that transfer by sensitized fluorescence can explain these results and that, therefore, they should not be used as proof for a special quality of proteins to act as conductors for energy.

${ }^{25}$ Th. Bücher and J. Kaspers, Biochim. et Biophys. Acta 1, 21 (1947). 